\title{
A CONVERSE TO THE HIGH INDICES THEOREM ${ }^{1}$
}

WALTER RUDIN

Suppose $\left\{\lambda_{n}\right\}$ is an increasing sequence of positive numbers which satisfies Hadamard's gap condition

$$
\liminf _{n \rightarrow \infty}\left(\lambda_{n+1} / \lambda_{n}\right)>1,
$$

and let $a_{n}$ be real numbers such that the series

$$
f(x)=\sum a_{n} x^{\lambda_{n}}
$$

converges for $0 \leqq x<1$. In this situation, the "high indices theorem" of Hardy and Littlewood [1], [2] asserts that the series $\sum a_{n}$ converges if $f(x)$ tends to a finite limit as $x \rightarrow 1$; analogously, Zygmund [3] has proved that $\sum\left|a_{n}\right|<\infty$ if $f$ is of bounded variation in $(0,1)$. It has apparently not been observed that both of these results belong to the rare type of gap theorem for which the known sufficient gap condition is also necessary:

Theorem. If $0<\lambda_{1}<\lambda_{2}<\cdots$, if $\lambda_{n} \rightarrow \infty$, and if

$$
\liminf _{n \rightarrow \infty}\left(\lambda_{n+1} / \lambda_{n}\right)=1
$$

then there is a function $f$ of the form (2) which is monotonic and bounded in the segment $(0,1)$ although the series $\sum a_{n}$ diverges.

Proof. By (3), $\left\{\lambda_{n}\right\}$ has a subsequence (again denoted by $\left\{\lambda_{n}\right\}$ ) such that $\lambda_{n}>n$ and

$$
\lambda_{2 k}<\left(1+k^{-3}\right) \lambda_{2 k-1} \quad(k=1,2,3, \cdots) .
$$

If $0<\alpha<\beta$ and $\beta / \alpha=1+1 / \gamma$, define

$$
g(x)=g(\alpha, \beta ; x)=x^{\alpha}-(\alpha / \beta) x^{\beta} .
$$

Then $g$ increases from 0 to $1 /(1+\gamma)$ as $x$ increases from 0 to 1 . Therefore (4) implies that the functions

$$
f_{1}(x)=\sum k g\left(\lambda_{2 k-1}, \lambda_{2 k} ; x\right) \text { and } f_{2}(x)=\sum g\left(\lambda_{2 k-1}, \lambda_{2 k} ; x\right)
$$

have the stated properties. Note that $f_{1}$ has unbounded coefficients $a_{k}$. The corresponding coefficients of $f_{2}$ are bounded but do not tend to 0 .

Received by the editors October 4, 1965.

${ }^{1}$ Research supported by NSFGP-3483. 


\section{REFERENCES}

1. G. H. Hardy and J. E. Littlewood, A further note on the converse of Abel's theorem, Proc. London Math. Soc. 25 (1926), 219-236.

2. A. E. Ingham, On the "high-indices" theorem of Hardy and Littlewood, Quart. J. Math. 8 (1937), 1-7.

3. A. Zygmund, On certain integrals, Trans. Amer. Math. Soc. 55 (1944), 170-204.

UNIVERSITY OF WISCONSIN

\section{REGULAR BOUNDARY POINTS IN MARKOV CHAINS}

\section{A. W. KNAPP 1}

1. Introduction. In classical potential theory [4], a boundary point $p$ of a bounded domain $D$ in Euclidean space is called regular if there is a continuous superharmonic function $h \geqq 0$ defined in $D$ which tends to zero at $p$ and which has a strictly positive lower bound outside any neighborhood of $p$. The tip of a Lebesgue spine is an example of a point which is not regular. The theorem is that the Dirichlet problem for $D$ is solvable for continuous boundary values if and only if every point of the boundary is regular. Moreover, the natural candidate for a solution approaches the given boundary values at every regular point.

The question arises as to the nature of the set of regular boundary points of a domain. It is known [2, p. 225] that this set is not empty and, in fact, that its complement in the boundary has exterior capacity zero.

The same question can be and has been asked for Markov chains. If $P$ is the transition matrix of a transient Markov chain, then a function (column vector) $h$ is harmonic if it has the mean value property $h=P h$, superharmonic if $h \geqq P h$. Doob [1] and Hunt [3] have developed a Martin boundary theory for such a chain, and they showed that, in terms of the space they construct, there is the usual sort of Poisson-integral representation theorem for the integrable nonnegative superharmonic functions. In this framework we ask two questions: Is the set of regular points a Borel set? Is the set neces-

Received by the editors August 2, 1965.

1 This research was supported by Air Force Office of Scientific Research Grant AF-AFOSR 335-63. 